\title{
Adult Onset Systemic Carnitine Deficiency: Favorable Response to L-Carnitine Supplementation
}

\author{
M.D. Levitan, J.T. Murphy, W.G. Sherwood, J. Deck and G.M.J. Sawa
}

\begin{abstract}
We report the case of a patient who at age 39 first developed an episode of muscle weakness and transient ketoacidosis with biopsy proven fatty infiltration of the liver. Over the next several years, myopathy ensued; biopsy revealed extensive deposition of lipid globules in type 1 muscle fibres. Further investigations established the diagnosis of systemic carnitine deficiency (SCD) with skeletal muscle, hepatic, and cardiac involvement. The patient has benefited from L-carnitine supplementation. Our case represents an unusually late onset of SCD and highlights the necessity, when appropriate, of a high index of suspicion of this rare but treatable disorder.
\end{abstract}

\begin{abstract}
RÉSUMÉ: Déficit systémique en carnitine apparu à l'âge adulte: réponse favorable suite à un apport complémentaire de L-carnitine Nous décrivons un patient qui, à l'âge de 39 ans, a présenté pour la première un épisode de faiblesse musculaire avec acido-cétose temporaire. La biopsie hépatique a révélé une infiltration graisseuse du foie. Dans les années subséquentes, une myopathie s'est installée; une biopsie a démontré la présence de globules lipidiques dans les fibres musculaires de type 1. Par la suite, ou a pu établir un diagnostic de déficience systémique en carnitine (DSC) qui atteignait les systèmes musculo-squelettique, cardiaque et hépatique. Le patient s'est amélioré à la suite de l'administration d'un supplément diététique de L-carnitine. Ce cas présente une DSC à début tardif et démontre bien l'importance de considérer, lorsque le tableau clinique le suggère, la possibilité que le patient souffre de cette condition rare, mais néanmoins traitable.
\end{abstract}

Can. J. Neurol. Sci. 1987; 14:50-54

Over the decade since its initial description by Karpati and co-workers, ' the syndrome of systemic carnitine deficiency has received much attention with regard to descriptions of its varied clinical presentations, ${ }^{2-8}$ treatment, ${ }^{9-11}$ and efforts to understand its basic pathophysiology. ${ }^{12-20}$ Typically, this disorder presents in childhood with recurrent episodes of encephalopathy simulating Reye's syndrome. An overabundance of lipid globules in skeletal and frequently, cardiac muscle cells as well as hepatocytes is a well recognized feature of this syndrome. There is usually a decrease in the level of serum carnitine. The precise etiolngy of SCD remains controversial. A number of reports have docimented dramatic improvement by L-carnitine supplementation. We describe an unusual case of SCD in which clinical manifestations of the disorder began in a man at age 39 .

\section{CASE RePORT}

This 45 year old physical education instructor was well until age 39 when he experienced a one week episode of nausea, vomiting, and fatigue. Laboratory tests showed metabolic acidosis. Recovery was spontaneous, however, one month later nausea and vomiting recurred and were accompanied by severe exertional fatigue. He complained of difficulty in walking which he attributed to bilateral leg weakness. Past medical history included duodenal ulcer disease and was negative both for ethanol abuse and diabetes mellitus. Family history revealed that his father had idiopathic spastic paraparesis with onset of ataxia in the fifth decade. There was no history of parental consanguinity or of neuromuscular disease in any of the patient's three offspring. Examination revealed a thin, dehydrated man with mild drowsiness and left upper quadrant abdominal tenderness. Metabolic acidosis with mild ketonemia and ketonuria were found (bicarbonate $11 \mathrm{mMol} / \mathrm{L}$, serum and urine ketones positive at 1:4 dilution). Serum glutamate oxaloacetate transaminase (SGOT) was elevated at 151 units/L (normal up to 40). Serum creatine kinase (CK) was not recorded. A liver-spleen scan revealed mild hepatomegaly with patchy uptake of colloid suggesting reticuloendothelial dysfunction. Hepatitis $B$ surface antigen was negative. Liver biopsy demonstrated moderate excess of lipid globules in hepatocytes (Figure 1). A small bowel biopsy was performed to investigate the possibility of malabsorption and was negative. The patient made a full recovery with rehydration and bicarbonate supplementation. A precise diagnosis was not made.

The patient then remained well until the age of 42 when repeated episodes of profound exertional fatigue recurred. Neurological examination revealed moderate weakness in neck flexors as well as in the proximal muscles of the upper and lower extremities (MRC grade 4-/5). The patient was unable to arise from the supine position without the use of his hands. The ankle tendon reflexes were normal. Cerebellar and sensory function were normal. Total CK was $1300(<250) \mathrm{u} / \mathrm{L}$. An

From The Toronto General Hospital, Divisions of Neurology and Neuropathology. Departments of Medicine, Pathology and The Hospital for Sick Children, Department of Pediatrics, University of Toronto

Received April 17. 1986. Accepted in final form November 4. 1986

Reprint requests to: Dr. J.T. Murphy, 11-221 Eaton North. Toronto General Hospital, 200 Elizabeth St., Toronto, Ontario. Canada M5G 2C4 
EMG revealed occasional low amplitude motor unit potentials in the right deltoid and was otherwise normal. A biopsy of the left quadriceps muscle was reported as normal. Episodes of weakness continued to recur over the next two years. During a severe attack the patient was unable to arise from the gymnasium floor without assistance. There was no history of myoglobinuria. He was readmitted for further investigation in February, 1985. A history of periodic, usually exertional chest discomfort was obtained. Examination revealed moderate muscle atrophy in the face and neck, as well as in the pelvic and shoulder girdles. Muscle weakness as recorded previously was noted. Cardiovascular examination revealed findings typical of a bicuspid aortic valve. Hepatomegaly was absent. SGOT and CK activities were 98 and 389 $\mathrm{u} / \mathrm{L}(<250)$. The CK MB isoenzyme fraction was $0.09(<0.04)$ suggesting myocardial as well as skeletal muscle disease. Serum lactate was normal. An echocardiogram demonstrated mild left ventricular hypokinesis and minimal aortic insufficiency. EMG findings included the presence of diffuse high amplitude polyphasic motor unit potentials of prolonged duration.

A left deltoid muscle biopsy showed preponderance of type 1 fibres which were of somewhat smaller average size than the type 2 fibres (Figure 2). The type 1 fibres contained numerous small vacuoles which proved on the oil red o stain to be filled with lipid. The lipid content of these fibres was far in excess of that normally present (Figure 3). Glycogen was present in normal amounts. Electron microscopy (Figure 4) showed the lipid vacuoles as rounded empty spaces lacking a limiting membrane and dispersed between myofibrils and beneath the plasma membrane. Mitochondria were increased in number and were often seen in clusters adjacent to the lipid vacuoles. Many of the mitochondria contained paracrystalline inclusions. Review of the muscle biopsy performed two years before showed slight microvacuolation of type 1

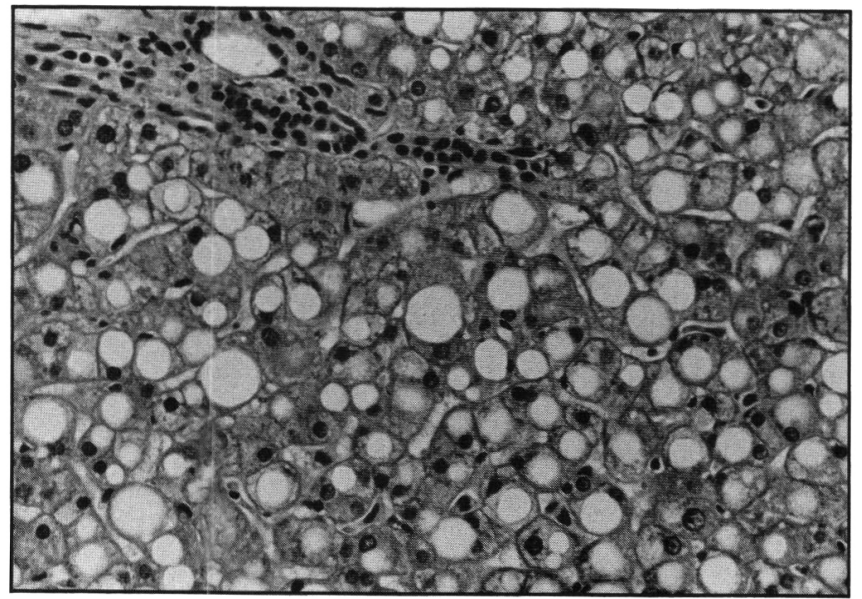

Figure 1 -Liver biopsy demonstrating lipid droplets distending hepatocytes. $H \& E \times 25$

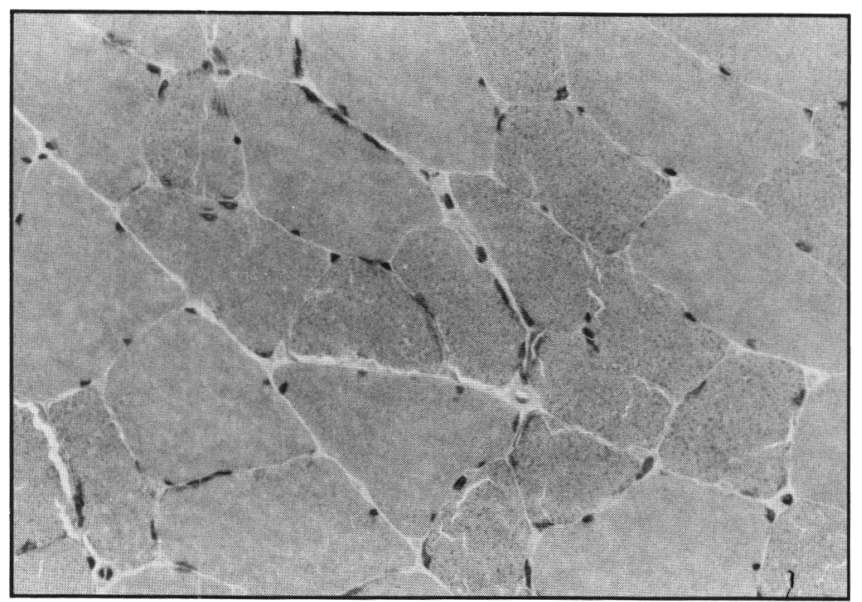

Figure $2-$ Left deltoid muscle biopsv of 1985. Scattered relatively small fibres demonstrate a diffuse pattern of basophilic stippling. ATPase stains proved these to represent type 1 fibres. $H \& E \times 25$ fibres, however fat stains and EMG had not been performed at that time. Electron micrographs prepared when this material was later examined showed a mild excess of mitochondria and lipid, but no paracrystalline inclusions were seen. These findings were minimal in comparison to those of the subsequent biopsy.

A tentative diagnosis of systemic carnitine deficiency was made. This was supported subsequently by a random measurement of serum free (unacylated) and total carnitine yielding a free/total ration of 22/83 (uMol/L), well below the normal ratio of $47 / 60 .{ }^{6}$ Several months later the patient was readmitted for a 48 hour caloric deprivation test for diagnostic confirmation prior to the commencement of treatment. Physical examination at that time was unchanged (Figure 5).

The patient underwent a 48 hour fast in hospital. This was well tolerated. Water was allowed ad libitum. Blood samples were drawn at 24 and 48 hours for determination of free and total carniline, 3-hydroxybutyrate, free fatty acids, lactate and glucose. A 24 hour urine collection half way into the fast was obtained for measurement of free and total carnitine and dicarboxylic acids. Serum determinations of glucose. lactate, free fatty acids and 3-hydroxybutyrate were performed by well established techniques. Carnitine levels were measured by radioisotopic assay as described by McGarry and Foster. ${ }^{21}$ Urinary organic acids were analyzed by gas chromatography-mass spectroscopy as described by Goodman and Markey. ${ }^{22}$ Results of the deprivation tests are shown in Table 1. A profound drop in the level of serum free carnitine occurred on day two of the fast. This was accompanied by a moderate rise in ketone body (hydroxybutyrate) production and a mild rise in serum lactate. Significant hypoglycemia occurred by the end of the fast. Urine screening revealed a large amount of free carnitine excretion.

Following diagnostic confirmation, L-carnitine supplement (1 gram three times a day with meals) was begun. Over the next several months the patient's muscle strength showed impressive improvement to grade $4+15$ and he has resumed teaching gymnastics. He has not reported any

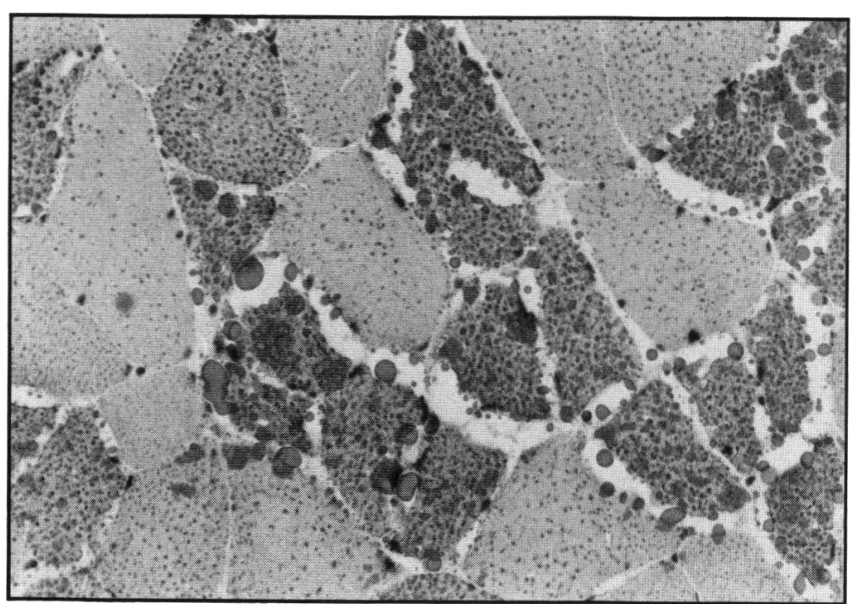

Figure $3-$ a) Oil Red 0 section demonstrating a marked excess of lipid showing as dark staining globules largely confined to type I muscle fibres. $\times 25$.

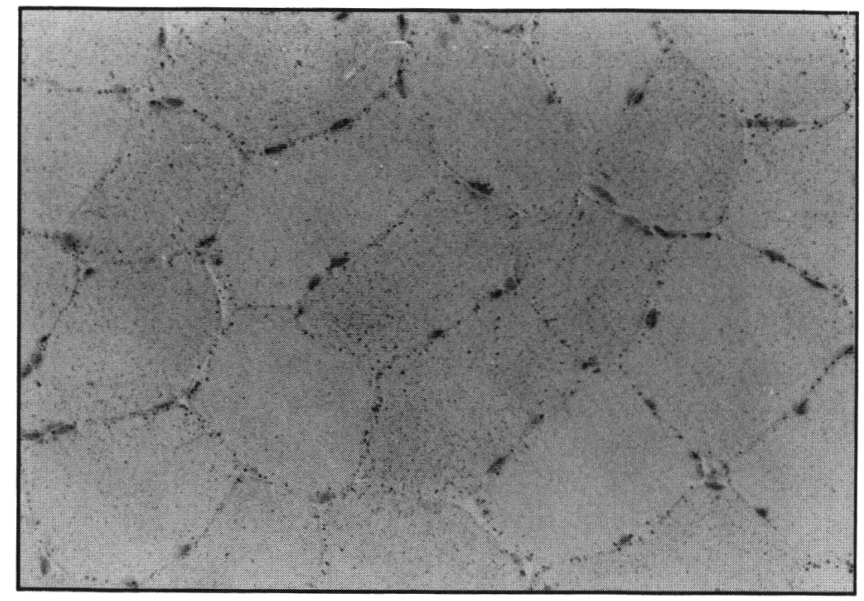

Figure $3-b)$ Normal control. Oil Red $0 \times 25$ 
significant side effects to treatment. A recent CK was normal ( $161 \mathrm{u} / \mathrm{L})$. His latest examination 18 months post initiation of therapy showed a 15 pound weight gain and normal muscle power throughout. Minimal shoulder atrophy was present and the deep tendon reflexes were all normal.

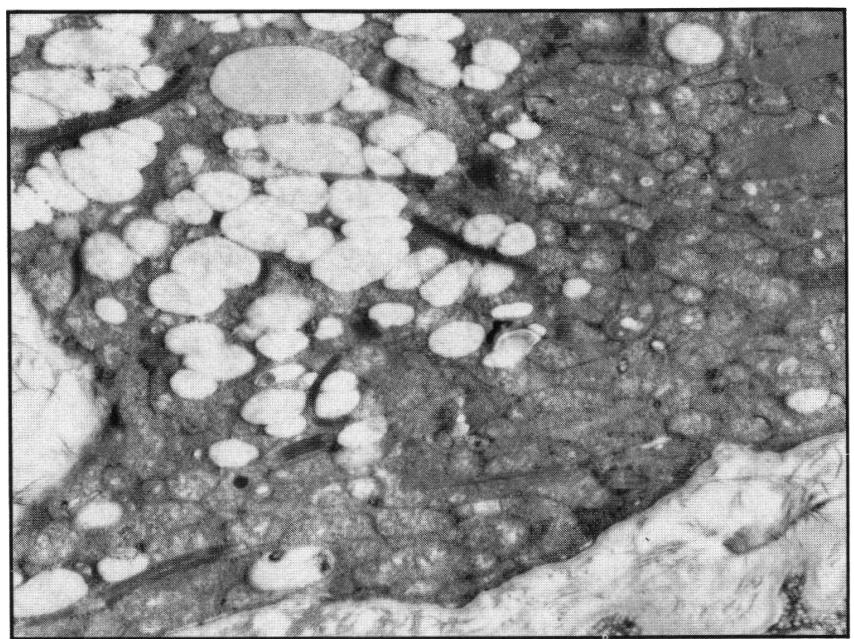

Figure 4 - a) Electron micrograph of 1985 deltoid muscle biopsy. Excessive mitochondria are seen packed beneath the plasma membrane. Some contain paracrystalline inclusions. Numerous pale fat droplets are also present. Original Mag. $x 7700$

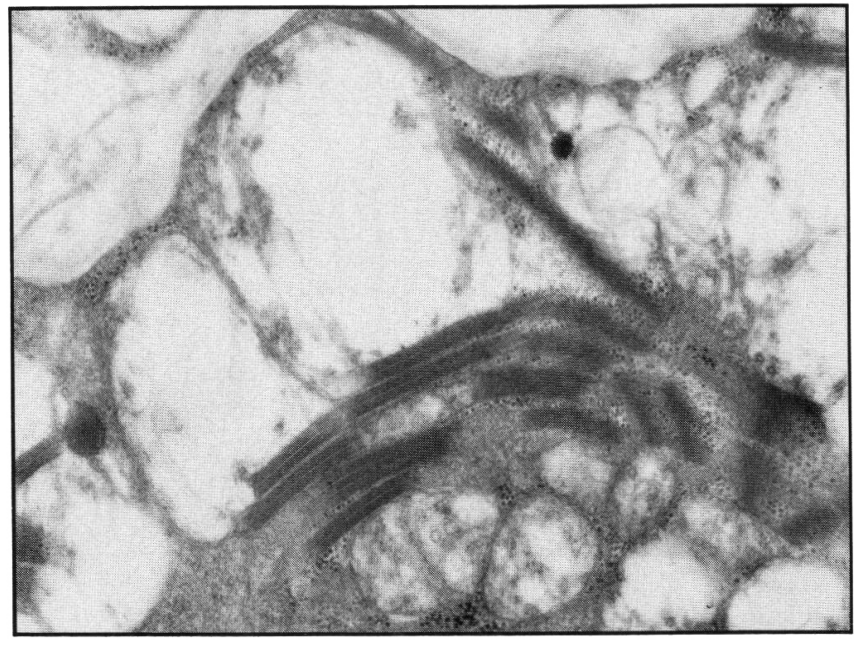

Figure $4-b)$ Higher magnification of intramitochondrial paracrystalline inclusions. Original Mag. × $31,500$.

\section{Discussion}

In 1975 Karpati and co-workers first described the entity of systemic carnitine deficiency in an 11 year old boy who originally presented with acute, transient hepatic insufficiency and encephalopathy.' Overt myopathic features occurred $11 \frac{1}{2}$ years later. Our patient's presentation bears similarity to this case. In particular, myopathic signs developed several years after the initial episode of liver dysfunction associated with lipid globule excess in hepatocytes. Such delays between initial features of SCD and myopathy have been reported. ${ }^{2.4} \mathrm{~A}$ "pre-myopathic" phase lasting between 2 and 10 years and characterized by recurrent episodes of weakness, nausea, vomiting and stupor has been described by Cornelio et al. ${ }^{4}$ Many case reports illustrate the variability with which SCD may present clinically and biochemically. ${ }^{2-8}$ For instance, recurrent episodes of non-ketotic hypoglycemia may present as the sole initial manifestation of SCD. ${ }^{5}$ Scarlato and coworkers have commented on the rarity of encephalopathy in adult onset SCD. ${ }^{3}$ Such diversity in expression awaits explanation.

Although uncommon, a number of cases of adult onset SCD have been reported. Scarlato et al described a man who at age 35 developed SCD characterized by diffuse muscle weakness and hepatic dysfunction. Plasma and muscle carnitine levels were depressed. Diagnosis was substantiated on liver and muscle biopsy. ${ }^{3}$ Markesbury and colleagues reported a 38 year old diabetic with onset of pelvic girdle weakness, generalized mus-

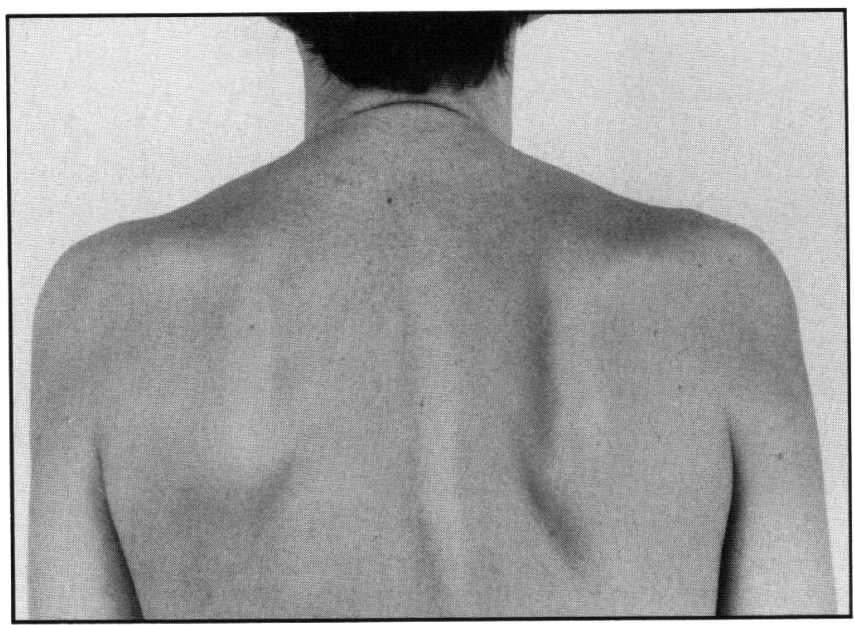

Figure 5-Posterior view showing shoulder girdle as well as infraspinatus muscle atrophy.

Table 1: Results of the 48 hour caloric deprivation test

\begin{tabular}{|c|c|c|c|c|c|}
\hline \multirow[t]{2}{*}{48 Hours } & $1.94(3.6+0.3)$ & $3.13(1.0+0.3)$ & $2.39(0.54+0.04)$ & $2.49(0.5+0.2)$ & $2 / 62(31 / 50)$ \\
\hline & & & & & $24(161+26)$ \\
\hline
\end{tabular}

24 Hour urine free carnitine excretion

Urine at 24 hours contained large amounts of 3-hydroxybutyric, acetic, and adipic acids and a moderate amount of suberic acid.

Serum values in parentheses represent control data derived by DiDonato et al ${ }^{14}$ from 4 adult males (age 20-40) with minor neurological disease unrelated to muscle pathology or metabolic disease.

Values aré accompanied by their respective standard deviations.

The 24 hour urinary carnitine (free) control value is from Carrier et a ${ }^{13}$ and was derived from 16 healthy older children and adults. 
cle atrophy, and areflexia. Lipid globule excess was demonstrated in muscle, leukocytes and Schwann cells. ${ }^{23}$ The latter finding may have contributed to the patient's areflexia and EMG findings of denervation. Our patient's EMG suggested denervation. Snyder et al have suggested that denervation changes on EMG in carnitine myopathy may simply reflect muscle membrane instability. ${ }^{24}$

Our patient demonstrated evidence of mild cardiomyopathy in addition to skeletal muscle and hepatic involvement. Prior to the advent of carnitine therapy, most patients with this constellation of features succumbed in childhood, usually from cardiac arrest. ${ }^{4}$ Post mortem examinations have revealed lipid globule accumulation and markedly depressed carnitine levels in skeletal and cardiac muscle as well as liver. ${ }^{2-4}$

Apart from induced ketosis, data regarding the biochemical sequelae of caloric deprivation in our patient are representative of those previously reported in carnitine myopathy. 1.5,14.21.26 Specifically, serum free carnitine fell drastically by 48 hours into the fast. A nearly identical result was noted by Carrol et al who in addition noted a substantially milder drop in serum free carnitine in fasted controls. ${ }^{25}$ Severe hypoglycemia may have been the result of an impairment of gluconeogenesis. The large amount of dicarboxylic acid excretion in the urine likely arose from the omega oxidation of free fatty acids which is carnitine independent and presumably occurs outside the mitochondrion.' Ketone body elevation is not anticipated in SCD since there is a defect in fatty acid oxidation. A possible explanation for the ketosis observed in this case is that during the fast, serum free carnitine fell due to its sequestration in liver, allowing for long chain fatty acid metabolism to occur. ${ }^{14}$ Alternatively, it is possible that medium chain fatty acids not requiring carnitine for their oxidation were used as an alternate source of energy production in the liver. ${ }^{27}$ A recent report has documented exaggerated ketogenesis occurring during fasting in cases of secondary carnitine deficiency associated with organic acidemia. ${ }^{28}$

As initially demonstrated by Karpati et al ${ }^{1}$ and further documented in subsequent case reports, ${ }^{3.9-11}$ oral carnitine supple-

Table 2: Secondary Carnitine Deficiency Syndromes

1. Secondary to inborn errors of metabolism methylmalonic aciduria isovaleric acidemia glutaric aciduria type 11 proprionic acidemia Kearnes Sayre syndrome

2. Secondary to other conditions chronic renal failure cirrhosis with malnutrition renal Fanconi syndrome valproate induced Reye's syndrome pregnancy

\footnotetext{
Table 3: Lipid Myopathy with Normal Muscle Carnitine

carnitine palmityle transferase deficiency (symptomatic phase) neutral lipid storage disease with ichthyosis viral hepatitis

mitochondrial myopathy*
}

mentation is highly effective in the treatment of SCD. These latter reports include cases of adult onset SCD. Clinical, biochemical and morphological responses to therapy are dramatic. In particular, muscle weakness and atrophy diminish markedly, as does the lipid globule excess in muscle. Serum levels of carnitine as well as liver enzymes return to normal.

Carnitine serves as a cofactor in the transfer of long chain fatty acids across the inner mitochondrial membrane in order for their $\beta$-oxidation to occur. ${ }^{6.7}$ Triglyceride accumulation in carnitine deficient tissues has been attributed to defective $\beta$-oxidation with subsequent esterification of unmetabolized fatty acids to glycerol.'

Traditionally, lipid myopathy associated with carnitine deficiency has been classified into two main varieties. A so-called "myopathic" form involves muscle weakness and decreased concentration of muscle carnitine with normal serum carnitine. In contrast, SCD is characterized by decreased carnitine levels in serum, muscle and liver, and usually manifests the additional feature of recurrent episodes of hepatic encephalopathy mimicking Reye's syndrome. A substantial degree of overlap exists between these two varieties. ${ }^{6.7}$ The mechanism underlying the "myopathic" form may involve defective transport into muscle. ${ }^{23}$ This form also responds to carnitine supplementation. ${ }^{7}$ With regard to $\mathrm{SCD}$, both the precise mechanism underlying the more widespread carnitine depletion as well as the intriguing intermittency of symptoms often noted await further clarification. A renal carnitine leak may be operative though such a leak may be asymptomatic. ${ }^{16}$ There have been no convincing reports indicating decreased synthesis or increased degradation of carnitine. ${ }^{15,17}$

Stumpf and co-workers have recently emphasized drawbacks in the simple classification of carnitine deficiency syndromes into myopathic and systemic varieties. They redefine carnitine deficiency as " a condition in which insufficient carnitine exists to buffer the toxic actions of acyl CoA metabolites". 12 Stress was placed on the importance of the ratio of acyl to free (active) carnitine rather than on simple absolute levels of carnitine. This argument reflects current speculation that SCD reflects a fundamental defect in fatty acid oxidation leading to a reduction in the ratio of free/acyl carnitine. For example, a number of conditions have been identified in which carnitine is depleted in association with inborn as well as acquired abnormalities of metabolism (Table 2). ${ }^{12}$ Furthermore, lipid myopathy may occur in the absence of carnitine deficiency (Table 3). ${ }^{6.12}$

Our patient clearly achieved benefit from L-carnitine supplementation. Although the precise mechanism underlying SCD awaits elucidation, diagnosis of this condition is essential given proven clinical, biochemical and histological response to therapy. Muscle biopsy is an essential step in arriving at this diagnosis.

\section{ACKNOWLEDGEMENTS}

The authors wish to thank Dr. G. Karpati for helpful comments in reviewing this manuscript, and Mrs. R. Abramovitch for assistance in its preparation. Assistance in translation of the abstract was kindly provided by Dr. J. Saint-Cyr. L-carnitine was kindly provided by Sigma Tau, Rome. Carnitine analyses were supported by funds from the Physicians Services of Toronto.

*Muscle carnitine may be slightly reduced 


\section{REFERENCES}

1. Karpati G, Carpenter S. Engel AG, et al. The syndrome of systemic carnitine deficiency. Neurology 1975; 25: 16-24.

2. Ware AJ, Burton WC, McGarry JD, et al. Systemic carnitine deficiency. Report of a fatal case with multisystemic manifestations. J Ped 1978: 93: 959-964.

3. Scarlato G, Pellegrini G, Cerri G, et al. The syndrome of systemic carnitine deficiency: Morphological and metabolic correlations in two cases. Can J Neurol Sci 1978; 5: 205-213.

4. Cornelio F, DiDonato S, Peluchetti D, et al. Fatal cases of lipid storage myopathy with carnitine deficiency. J Neurol Neurosurg Psych 1977: 40: 170-178.

5. Slonin AE, Borum PR, Mrak RE, et al. Nonketotic hypoglycemia: An early indicator of systemic carnitine deficiency. Neurology 1983: 33: 29-33.

6. Rebouche CJ, Engel AG. Carnitine metabolism and deficiency syndromes. Mayo Clin Proc 1983; 58: 533-540.

7. Dimauro S, Trevisan C. Hays A. Disorders of lipid metabolism in muscle. Muscle and Nerve 1980; 3: 369-383.

8. Tripp ME, Katcher ML, Peters HA, et al. Systemic carnitine deficiency presenting as familial endocardial fibroelastosis. New Eng J Med 1981; 305: 385-390.

9. DiDonato S, Peluchetti D. Rimoldi M, et al. Systemic carnitine deficiency: clinical, biochemical and morphological cure with L-carnitine. Neurology 1984; 34: 157-162.

10. Chapoy PR, Angelini C, Brown WF, et al. Systemic carnitine deficiency - A treatable inherited lipid storage disease presenting as Reye's syndrome. New Eng J Med 1980; 303: 1389-1394.

II. Waber LJ, Valle D, Neill C, et al. Carnitine deficiency presenting as familial cardiomyopathy: A treatable defect in carnitine transport. J Ped 1982; 101: 700-705.

12. Stumpf DA. Parker WD, Angelini C. Carnitine deficiency, organic acidemias, and Reye's syndrome. Neurology 1985;35: 1041-1045.

13. Carrier HN. Berthillier G. Carnitine levels in normal children and adults and in patients with diseased muscle. Muscle and Nerve 1980; 3: 326-334.
14. DiDonato S, Cornelio F. Storchi G, et al. Hepatic ketogenesis and muscle carnitine deficiency. Neurology 1979; 29: 780-785.

15. Rebouche CJ, Engel AG. Primary systemic carnitine deficiency: 1 . Carnitine biosynthesis. Neurology 1981:31: 813-818.

16. Engel AG, Rebouche CJ. Wilson DM, et al. Primary systemic carnitine deficiency 11. Renal handling of carnitine. Neurology 1981; 31: 819-825.

17. Rebouche CJ, Engel AG. In vitro analysis of hepatic carnitine biosynthesis in human systemic carnitine deficiency. Clin Chim Acta 1980; 106: 295-300.

18. Rebouche CJ, Engel AG. Kinetic compartment analysis of carnitine metabolism in the human carnitine deficiency syndromes. J Clin Inv 1984:857-867.

19. Willner JH, Ginsburg S, Dimauro, S. Active transport of carnitine into skeletal muscle. Neurology 1978; 28: 721-724.

20. Engel AG, Angelini C. Carnitine deficiency of human skeletal muscle with associated lipid storage myopathy: A new syndrome. Science 1973; 179: 899-902.

21. McGarry JD, Foster DW. An improved and simplified radioisotopic assay for the determination of free and esterified carnitine. J Lipid Res 1976; 17: 277-280.

22. Goodman SI, Markey GP. Diagnosis of organic acidemias by gas chromatography-mass spectrometry. New York, Allan R. Liss, 1981; I05-114.

23. Markesbery WR, McQuillen MP, Procopis PG, et al. Muscle carnitine deficiency. Arch Neurol 1974; 31: 320-324.

24. Snyder TM, Little BW, Roman-Campos G, et al. Successful treatment of familial idiopathic lipid storage myopathy with L-carnitine and modified lipid diet. Neurology 1982; 32: 1106-1115.

25. Carroll JE, DeVivo DC, Brooke MH, et al. Fasting as a provocative test in neuromuscular diseases. Metabolism 1979:28:683-687.

26. Carroll JE, Brooke MH, Shumate JB, et al. Carnitine intake and excretion in neuromuscular diseases. Am J Clin Nutr 1981: 34: 2693-2698.

27. Stryer L. Biochemistry. San Francisco. W.H. Freeman \& Co. 1975; pp 411.

28. Wolff JA, Thuy LP, Haas R, et al. Carnitine reduces fasting ketogenesis in patients with disorders of proprionate metabolism. Lancet 1986; Feb. 8, 289-291. 